\title{
Automated Event Detection of Epileptic Spikes using Neural Networks
}

\author{
Payal Khanwani \\ Medical Electronics \\ BMS College of Engineering \\ Bangalore, 560019
}

\author{
Susmita Sridhar \\ Medical Electronics \\ BMS College of Engineering \\ Bangalore, 560019
}

\author{
Mrs.K.Vijaylakshmi \\ Assistant Professor \\ Medical Electronics \\ BMS College of Engineering \\ Bangalore, 560019
}

\begin{abstract}
Biomedical signals carry signatures of physiological events. The part of the signal related to specific event is called epoch. Epilepsy is one of the important brain disorders which can be diagnosed and monitored is characterized by sudden recurrent and transient disturbances of mental function and movements of body which is caused from excessive discharge of brain cell groups. This excessive discharge is shown in EEG as epileptic spikes which are complementary source of information in diagnosis and localization of epilepsy. Artificial Neural networks have been provided an effective approach for a broad spectrum of applications for EEG signals because of its self-adaption and natural way to organize and implement the redundancy. It is well known that back-propagation networks are very suitable for pattern recognitions. The algorithm tested on 100 normal and abnormal datasets showed expected classification.
\end{abstract}

\section{General Terms}

Pattern Recognition, Neural Networks

\section{Keywords}

Epoch, Epilepsy, EEG, Artificial Neural Networks, BackPropagation

\section{INTRODUCTION}

Epilepsy is a symptom of paroxysmal and abnormal discharges in the brain that may be induced by a variety of pathological processes of genetic or acquired origin disturbances of mental function and/or movements of different body parts that result from excessive discharges of groups of brain cells. This excessive and hyper-synchronous activity of the brain is termed as a 'seizure'. Depending on the extent of involvement of the brain areas, divided into two main classes. Generalized seizures involve almost the entire brain where as focal (or partial) seizures originate from circumscribed regions of the brain and remain restricted to that region.

The definition of an epileptic spike is given by the committee on Terminology of International Federation of Societies for Electroencephalography and clinical neurophysiology is as follows: "... an EEG spike is a transient waveform, clearly distinguished from background activity with a pointed peak and duration from 20-70 ms". The epileptic waves have various morphologies with main ingredients like spikes $(12.5-50 \mathrm{~Hz})$, sharp $(5-12.5 \mathrm{~Hz})$, slow $(1-2.5 \mathrm{~Hz})$ waves.
Usually spikes and sharp waves (SSW) or sharp and slow (SWW) appear simultaneously.

Visual analysis of EEG is the most widespread of clinical EEG analysis. However, detecting and classifying sharp transient of the EEG record by visual screening is a laborious and time consuming task. In many cases the patients themselves are not aware of the occurrence of their own seizures. Thus there is a need to construct an algorithm for automated detection of epileptic spikes that makes detection based on objective criteria. The use of artificial neural networks and back propagation algorithm gives a promising answer to that need.

\section{MATERIAL AND METHODS \\ 2.1 Data Collection}

These data sets were collected from Department of Information and Communication Technologies Universitat Pompeu Fabra, Barcelona.60 data sets of epileptic and non epileptic data were used for training the neural network and 100 data sets for testing. Normal data set comprises of EEG recordings from healthy volunteers using standardized electrode placement scheme (Fig1.)The volunteers were relaxed with eyes open. The epileptic dataset contains only seizure activity. All EEG signals were recorded with the same 128-channel amplifier system. The sampling rate of the EEG signals is $173.61 \mathrm{~Hz}$. The time series have the spectral bandwidth of the acquisition system, which is $0.5 \mathrm{~Hz}$ to 85 $\mathrm{Hz}$. Band-pass filter settings were $0.53-40 \mathrm{~Hz}(12$

$\mathrm{dB} /$ oct.).Each data set consists of 4096 samples of one EEG time series.

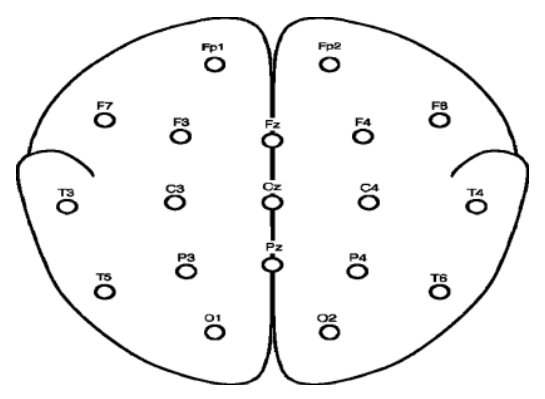

Figure 1. Scheme of the locations of surface electrodes according to the international $10-20$ system. 

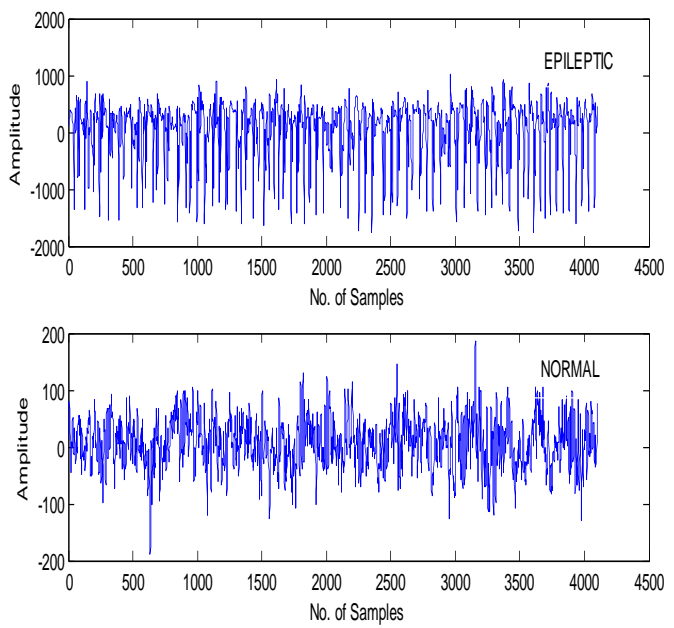
Signal

Figure 2.Plot of Normal and Epileptic

Figure 2 shows the plot of a normal data set obtained from healthy volunteers and epileptic seizure data set obtained from epileptogenic zones from epileptic patients.

\subsection{Feature Selection}

The following statistical features of the EEG are extracted as the input of neural network for spike detection:

- Minimum Amplitude

- Maximum Amplitude

- Mean

- Median

- Mode

- Standard Deviation

- Range

\subsection{Back-propagation Neural Network}

A two layered feed forward back-propagation network is used to classify the EEG signals. Selected features from the EEG are used to train and test the network for classifications of normal and seizure patterns. A Multi layer perceptron (MLP) is used because it is appropriate for solving pattern classification problems where supervised learning is implemented using back propagation algorithm (Fig 3). The advantage of using this type of ANN is rapid execution of trained network which is advantageous in signal processing applications. The back- propagation algorithm is as follows:

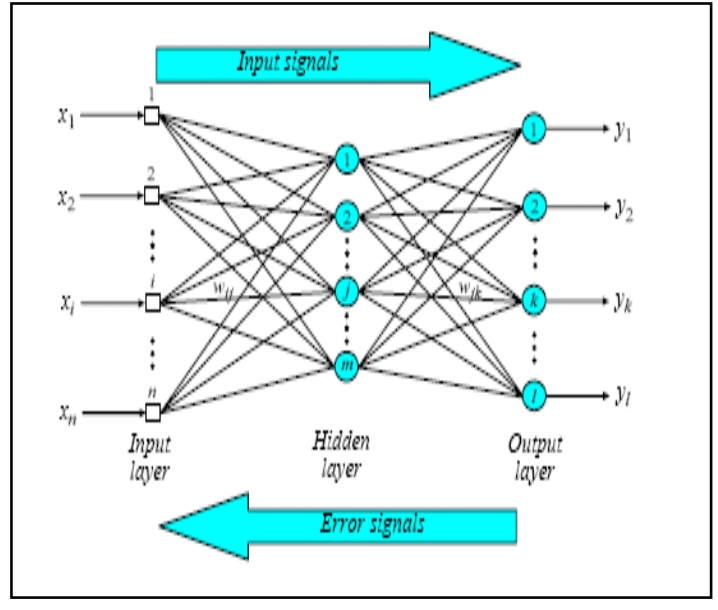

Figure 3.Back-propagation Neural Network

The activation function of the artificial neurons in ANNs implementing the back-propagation algorithm is a weighted sum (the sum of the inputs xi multiplied by their respective weights wji):

$$
A_{j}(\bar{x}, \bar{w})=\sum_{i=0}^{n} x_{i} w_{j i}
$$

The most common output function is the sigmoidal function:

$$
O_{j}(\bar{x}, \bar{w})=\frac{1}{1+e^{A_{i}(\bar{x}, \bar{w})}}
$$

The sigmoidal function is very close to one for large positive numbers, 0.5 at zero, and very close to zero for large negative numbers. This allows a smooth transition between the low and high output of the neuron (close to zero or close to one). We can see that the output depends only in the activation, which in turn depends on the values of the inputs and their respective weights. We can define the error function for the output of each neuron:

$$
E_{j}(\bar{x}, \bar{w}, d)=\left(O_{j}(\bar{x}, \bar{w})-d_{j}\right)^{2}
$$

The error of the network will simply be the sum of the errors of all the neurons in the output layer:

$$
E(\bar{x}, \bar{w}, \bar{d})=\sum_{j}\left(O_{j}(\bar{x}, \bar{w})-d_{j}\right)^{2}
$$


After we find this, we can adjust the weights using the method of gradient descendent:

$\Delta w_{j i}=-\eta \frac{\partial E}{\partial w_{j i}}$

To calculate how much the error depends on the output, which is the derivative of $\mathrm{E}$ with respect to $\mathrm{Oj}$ (from (3)) and how much the output depends on the activation, which in turn depends on the weights (from (1) and (2)):

$\frac{\partial E}{\partial O_{j}}=2\left(O_{j}-d_{j}\right)$

$\frac{\partial O_{j}}{\partial w_{j i}}=\frac{\partial O_{j}}{\partial A_{j}} \frac{\partial A_{j}}{\partial w_{j i}}=O_{j}\left(1-O_{j}\right) x_{i}$

From (6) and (7)

$\frac{\partial E}{\partial w_{j i}}=\frac{\partial E}{\partial O_{j}} \frac{\partial O_{j}}{\partial w_{j i}}=2\left(O_{j}-d_{j}\right) O_{j}\left(1-O_{j}\right) x_{j}$

The adjustment to each weight will be (from (5) and (8)):

$$
\Delta w_{j i}=-2 \eta\left(o_{j}-d_{j}\right) O_{j}\left(1-o_{j}\right) x_{i}
$$

Each iteration in back-propagation algorithm consists of 2 sweeps: forward activation to produce a solution and backward propagation of the computed error to modify the weights. These forward and backward sweeps continue until the ANN solution agrees with the desired value within a pre-specified tolerance. The non linear behavior of the back-propagation network allows the perceptron to generate complex decision regions, which is a desirable property in pattern classification.

\subsection{Methodology and Result}

Table 1: Training and Testing Patterns

\begin{tabular}{|l|l|}
\hline $\begin{array}{c}\text { No. of training } \\
\text { samples }\end{array}$ & 60 \\
\hline $\begin{array}{l}\text { No. of Epileptic } \\
\text { training samples }\end{array}$ & 30 \\
\hline $\begin{array}{l}\text { No. of Normal } \\
\text { training samples }\end{array}$ & 30 \\
\hline $\begin{array}{l}\text { No. of testing } \\
\text { patterns }\end{array}$ & 100 \\
\hline
\end{tabular}

The same neural network was trained using 60 data sets and tested using a new set of 100 epileptic and non epileptic data. A multilayer network was used with neurons or processing elements having a sigmoid transfer function. Input and target vectors were used to train the network until the network classified the input vectors correctly. With a learning rate of 0.05 and with a goal (acceptable error) of 1e-5 excellent results were obtained. The smaller we make the learning rate parameter, smaller will be the changes in the synaptic weights from one iteration to another and smoother will be the trajectory in weight space. The network exhibited expected generalization i.e. the network classified properly those inputs which were not used for training. The performance goal was met for 10 epochs in duration of 1.1513 seconds.

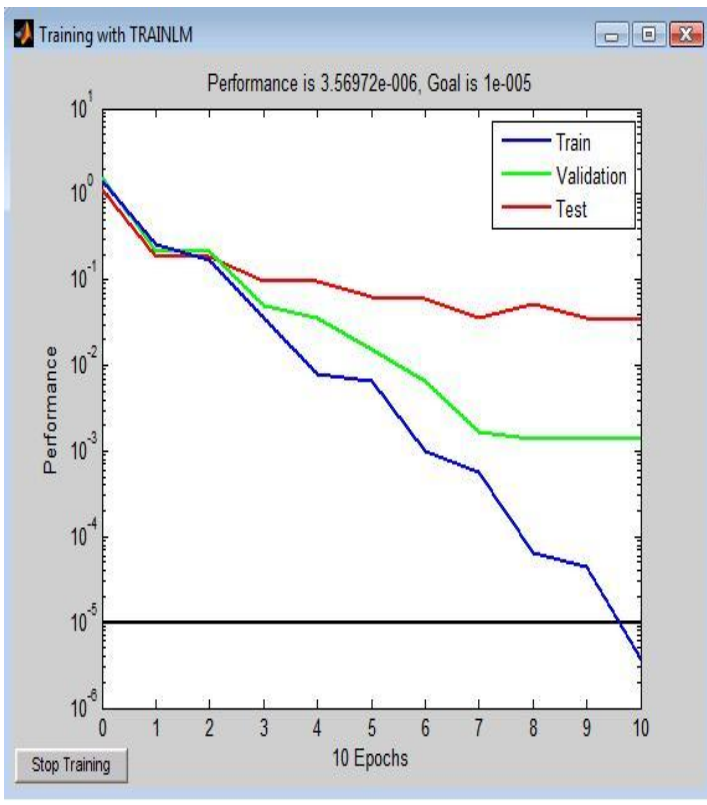

Figure 4.Training of designed Back-propagation Network

\subsection{Further Analysis and Graphical User Interface}

A graphical user interface (GUI) (figure.5) is created to make automated detection of epileptic spikes user friendly and to enable easier analysis. 


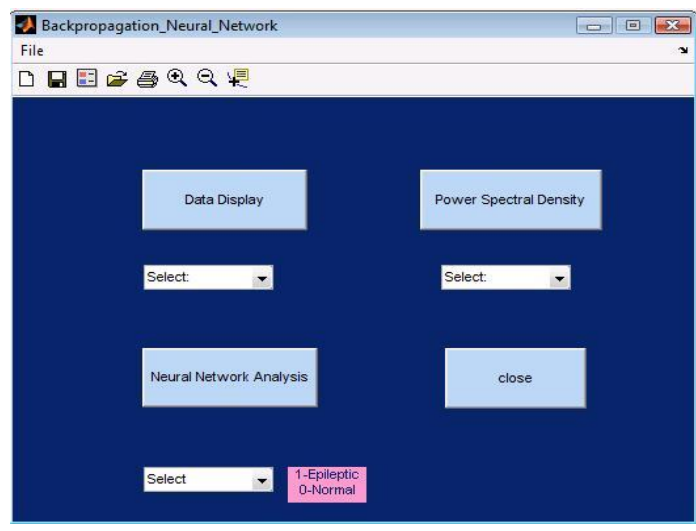

Figure 5. Graphical User Interface

\section{CONCLUSION}

A neural network is a massively parallel distributed processor made up of simple processing units which have the natural propensity for storing experiential knowledge through a learning process and making it available for use.

Despite the computational complexity involved, artificial neural networks offer several advantages in pattern recognition and classification in the sense of emulating adaptive human intelligence to a small extent. A simplistic approach for recognition of Epileptic spikes using artificial neural networks (ANN) has been described. Epilepsy is one of the most common of the serious neurological disorders characterized by unprovoked seizures. Hence automated detection is a dominant tool in Epilepsy diagnosis. Back propagation neural network serves as a suitable detector for classification of EEG signals and detection of Spike and Sharp Waves with excellent generalization as it provides an "approximation" to the trajectory in the weight space computed by method of steep descent.

\section{REFERENCES}

[1] "Epileptic Siezure Detection”,K.P Nayak,UC Niranjan,IFMBE Proceedings Vol 21,Kuala Lampur International Conference on Biomedical Engineering,Malaysia.

[2] "Neural network for automatic detection of EEG spikes" Shinn-Yih Tseng*, Fok-Ching Chong*, Rong-Chi Chen** and Te-Son Kuo*+, Proceedings of the 20th Annual International Conference of the IEEE Engineering in Medicine and Biology Society, Vol. 20, No 4,1998

[3] Automatic Detection of Epileptiform Events in EEG by a Three-Stage Procedure Based on Artificial Neural Networks Nurettin Acir*, 'Ibrahim Öztura, Mehmet Kuntalp, Barıs, Baklan, and Cüneyt Güzeli, IEEE Transactions On Biomedical Engineering, VOL. 52, NO. 1, January 2005.

[4] "Neural Networks- A comprehensive foundation, $2^{\text {nd }}$ Ed.”, Simon Haykin, Prentice Hall, 1998

[5] "Event Related Potentials Extraction from EEG Using Artificial Neural Network", Liu Mingyu, Ji Hongbing, Zhao Chunhong, 2008 Congress on Image and Signal Processing.

[6] G. Jando, R. M. Siegel, Z. Horvath, and G. Buzsaki, "Pattern recognition of the electroencephalogram by artificial neural networks," Electroenceph. Clinical Neurophysiology, vol. 86, no. 2, pp. 100-109, 1993.

[7] Biomedical Signal Analysis: A Case-Study Approach, Rangaraj M Rangayyan.

[8] Introduction to Neural Networks, Ben Krose, Patrick Van der Smagt, Eighth edition, November 1996. 